WHOI $-8 \mathrm{I}-24$

Woods Hole

copy 1

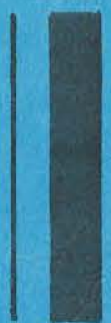

Oceanographic

Institution
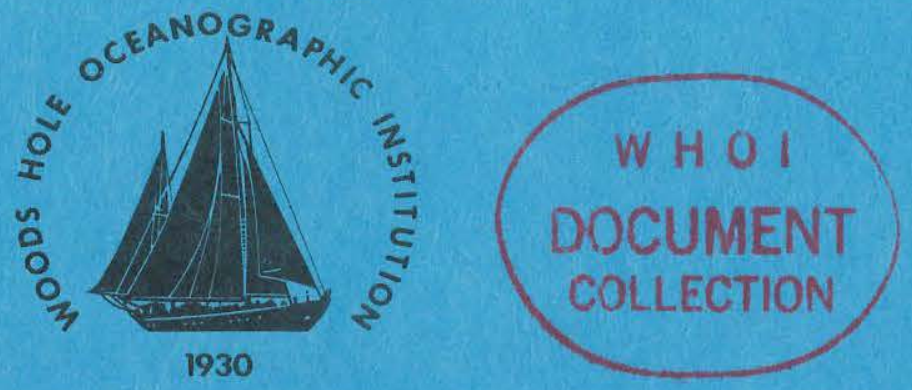

A TECHNIQUE FOR MEASURING THE PLANE-WAVE REFLECTION COEFFICIENT OF THE OCEAN BOTTOM

by

G.V. Frisk, A.V. Oppenheim, and D.R. Martinez

Apri1 1981

TECHNICAL REPORT

Prepared for the Office of Naval Research under Contracts N00014-77-C-0196 and N0001475-C-0951; NR 049-328.

Approved for public release; distribution unlimited.

IVOODS HOLE, MASSACHUSETTS 02543 
WHOI $-81-24$

A TECHNIQUE FOR MEASURING THE PLANE-WAVE REFLECTION COEFFICIENT OF THE OCEAN BOTTOM

by

\author{
G.V. Frisk, A.V. Oppenheim, \\ and D.R. Martinez \\ WOODS HOLE OCEANOGRAPHIC INSTITUTION \\ Woods Hole, Massachusetts 02543
}

Apri1 1981

Prepared fo: the Office of Naval Research under Contracts N00014-77-C-0196 and N00014-75-C-0951; NR 049-328.

Reproduction in whole or in part is permitted for any purpose of the United States Govermment. In citing this report in a bibliogrophy, the reference given should be to: J. Acoust. Soc. Am. 68 (2): 602-612. (August 1980).

Approved for public release; distribution unlimited.

Approved for Distribution: Earl E. Hays, Chairman Department of Ocean Encineering 


\title{
A technique for measuring the plane-wave reflection coefficient of the ocean bottom
}

\author{
George V. Frisk \\ Woods Hole Oceanographic Institution, Woods Hole, Massachusetts 02543
}

\begin{abstract}
Alan V. Oppenheim
Research Laboratory of Electronics, Massachusetts Institute of Technology, Cambridge, Massachusetts 02139
\end{abstract}

\author{
David R. Martinez ${ }^{\mathrm{a})}$ \\ MIT/WHOI Joint Program, Oceanography/Oceanographic Engineering, Woods Hole, \\ Massachusetts 02543
}

(Received 20 August 1979; accepted for publication 8 May 1980)

\begin{abstract}
A new technique for the measurement of the plane-wave reflection coefficient of a horizontally stratified ocean bottom is described. It is based on the exact Hankel transform relationship between the reflection coefficient and the bottom reflected field due to a point source. The method employs a new algorithm for the numerical evaluation of the Hankel transform which is based on the "projection-slice" theorem for the two-dimensional Fourier transform. The details of the algorithm are described in the companion paper. Although the algorithm is applied to the case of an isovelocity ocean, the general theory for measuring the plane-wave reflection coefficient in a refracting ocean is developed. The technique provides information about the reflection coefficient, not only for real incident angles, but also for complex angles, thus potentially providing substantial additional structural information about the bottom. The method is shown to yield excellent results with synthetically generated data for the cases of a hard bottom and slow isovelocity bottom.
\end{abstract}

PACS numbers: 43.30.Dr

\section{INTRODUCTION}

In a model of ocean acoustic propagation which assumes horizontal stratification, the plane-wave reflection coefficients of the surface and bottom as a function of horizontal wavenumber and frequency contain all the information about the boundaries necessary for the solution of acoustic problems in the water column. The assumption of horizontal stratification requires that the acoustic properties depend spatially only on depth. While the surface is suitably approximated as a pressure-release interface, the bottom cannot be generally modeled as an impenetrable boundary. In general the bottom may support both compressional and shear waves and may have properties which are frequency dependent (e.g. absorption). The reflection coefficient of the bottom is a complex-valued function with magnitude and phase variations. ${ }^{1}$ For a source at finite distance from the boundary, the behavior of the reflection coefficient must in general be known for all horizontal wavenumbers in order to compute accurately the total acoustic field. ${ }^{2}$ This implies that the reflection coefficient must be known for both real $(0 \leqslant \theta<\pi / 2)$ and complex $(\theta=\pi / 2-i \alpha, \alpha>0)$ incident angles, although it is conventially studied only at the real angles.

If one assumes a multilayered bottom with specific acoustic parameters, then one can compute the reflection coefficient by solving the wave equation in each layer and imposing continuity conditions at the layer interfaces. An example of this approach and a review of the literature in this area are provided by

\footnotetext{
a) Present address: Atlantic Richfield Company, Dallas, TX 75221.
}

the work of Hawker and Foreman. ${ }^{3}$ These methods are particularly useful in studying the effects of different bottom configurations on the behavior of the reflection coefficient. They can also be used to determine the reflection coefficient in a particular bottom area for which the input parameter data are known.

In this paper we address the problem of measuring the reflection coefficient, making only the assumption of horizontal stratification. This method relies on correctly relating the bottom reflected field data to the plane-wave reflection coefficient. Since acoustic sources do not radiate a single plane wave and the water column is in general an inhomogeneous medium, this is not a trivial matter. If the relationship is improperly made, one obtains unphysical results such as reflection coefficients which depend on source-receiver geometry or those which have values greater than unity for real incident angles. ${ }^{4,5}$ This paper describes a measurement technique which is based on the exact Hankel transform relationship between the reflection coefficient and the bottom reflected field due to a point source. The method employs a new algorithm for the numerical evaluation of the Hankel transform which is based on the "projection-slice" theorem for the two-dimensional Fourier transform. The algorithm is described in detail in the companion paper. ${ }^{6}$ The technique yields information about the reflection coefficient not only for real incident angles, but also for complex angles, thus potentially providing substantial additional information about the structure of the bottom. This is not possible with conventional measurement techniques. We show here that the method yields excellent results with synthetically generated data for the examples of a hard bottom and slow isovelocity fluid bottom. 
In Sec. I we review the general formalism within which plane-wave reflection coefficients arise in ocean acoustic propagation even in the case of an inhomogeneous ocean. In Sec. II, we describe the principle in measuring the reflection coefficient of the ocean bottom. In Sec. III, we present the application of our Hankel transform algorithm to the problem of measuring the reflection coefficient in an isovelocity ocean. The method is then applied to the examples of a hard bottom and slow isovelocity fluid bottom.

\section{THE THEORY OF ACOUSTIC PROPAGATION IN A HORIZONTALLY STRATIFIED OCEAN USING HANKEL TRANSFORMS}

For harmonic time dependence $[\exp (-i \omega t)]$ and a water column with sound speed $c(\mathbf{r})$, thickness $h$, and constant density, the spatial part of the acoustic pressure field satisfies the inhomogeneous Helmholtz equation

$$
\left[\nabla^{2}+k^{2}(\mathbf{r})\right] \mathrm{p}(\mathbf{r})=-4 \pi f(\mathbf{r}),
$$

where $k(\mathbf{r})=\omega / c(\mathbf{r})$. Using the formalism of Green's functions, ${ }^{7}$ we can in principle solve the problem for an arbitrary source distribution $f(\mathbf{r})$ and homogeneous boundary conditions if we know the solution $G$ for an impulse at $r=r_{0}$ corresponding to the solution of the equation

$$
\left[\nabla^{2}+k^{2}(\mathbf{r})\right]_{G}\left(\mathbf{r}, \mathbf{r}_{0}\right)=-4 \pi \delta\left(\mathbf{r}-\mathbf{r}_{0}\right) .
$$

If we assume horizontal stratification and cylindrical symmetry with the source at $r=0$ and $z=z_{0}$ as illus trated in Fig. 1, then Eq. (2) becomes

$\left[\frac{1}{r} \frac{\partial}{\partial r}\left(r \frac{\partial}{\partial r}\right)+\frac{\partial^{2}}{\partial z^{2}}+k^{2}(z)\right] G\left(r, z, z_{0}\right)=-2 \frac{\delta(r)}{r} \delta\left(z-z_{0}\right)$.

As the first step in solving this equation, we take the zero-order Hankel transform in $r$ of both sides, where the Hankel transform pair is defined as

$$
\begin{aligned}
& H\{F(r)\}=f\left(k_{r}\right)=\int_{0}^{\infty} r d r J_{0}\left(k_{r} r\right) F(r), \\
& H\left\{f\left(k_{r}\right)\right\}=F(r)=\int_{0}^{\infty} k_{r} d k_{r} J_{0}\left(k_{r} r\right) f\left(k_{r}\right) .
\end{aligned}
$$

Here $J_{0}$ is the zero-order Bessel function and $k_{r}$ is the horizontal wavenumber. Equation (3) then becomes

$$
\left(\frac{d^{2}}{d z^{2}}+k^{2}(z)-k_{r}^{2}\right) g\left(k_{r}, z, z_{0}\right)=-2 \delta\left(z-z_{0}\right),
$$

where $g\left(k_{r}, z, z_{0}\right)$ is the Hankel transform of $G\left(r, z, z_{0}\right)$ and we have used the relation

$$
H\left\{\frac{1}{r} \frac{d}{d r}\left(r \frac{d}{d r}\right) F(r)\right\}=-k_{r}^{2} f\left(k_{r}\right) .
$$

The depth-dependent Green's function ${ }^{8} g$ is given by

$$
\begin{aligned}
& g\left(k_{r}, z, z_{0}\right)=-\frac{2}{W\left(z_{0}\right)} p_{B}\left(k_{r}, z\right) p_{S}\left(k_{r}, z_{0}\right), \quad 0 \leqslant z \leqslant z_{0}, \\
& g\left(k_{r}, z, z_{0}\right)=-\frac{2}{W\left(z_{0}\right)} p_{B}\left(k_{r}, z_{0}\right) p_{S}\left(k_{r}, z\right), z_{0} \leqslant z \leqslant h,
\end{aligned}
$$

where the Wronskian $W\left(z_{0}\right)$ is

$$
W\left(z_{0}\right)=p_{B}\left(z_{0}\right) p_{S}^{\prime}\left(z_{0}\right)-p_{B}^{\prime}\left(z_{0}\right) p_{S}\left(z_{0}\right) .
$$

Here $p_{B}$ and $p_{S}$ are linearly independent solutions of the homogeneous form of Eq. (5) and satisfy homogeneous boundary conditions at the bottom and surface, respectively. These conditions can be expressed as the impedance relations

$$
\begin{aligned}
& p_{B} / \frac{\partial p_{B}}{\partial z}=\gamma_{B}, \quad \text { at } z=0, \\
& p_{S} / \frac{\partial p_{S}}{\partial z}=\gamma_{S}, \quad \text { at } z=h .
\end{aligned}
$$

The input parameters $\gamma_{B}$ and $\gamma_{S}$ contain the information about the boundaries necessary for the calculation of $g$. They depend on horizontal wavenumber and frequency, and furthermore $\gamma_{B}\left(\gamma_{S}\right)$ depends on the acoustic properties of the bottom (surface). Relative to the water column, they depend on the fluid loading at each interface, but are independent of the functional forms of the sound speed profile $c(z)$ and the solutions $p_{B}$ and $p_{S}$. Thus $\gamma_{B}$ depends on the water density and sound speed $c_{B}$ at the water-bottom interface, while $\gamma_{S}$ depends on the water density and sound speed $c_{S}$ at the water-surface interface (c.f. Fig. 1). We can therefore rewrite the condition in Eq. (8a) in terms of the plane wave reflection coefficient $R_{B}$ associated with a half-space of sound speed $c_{B}$ bounding the bottom. Similarly, we can rewrite the condition in Eq. (8b) in terms of the plane-wave reflection coefficient $R_{S}$ associated

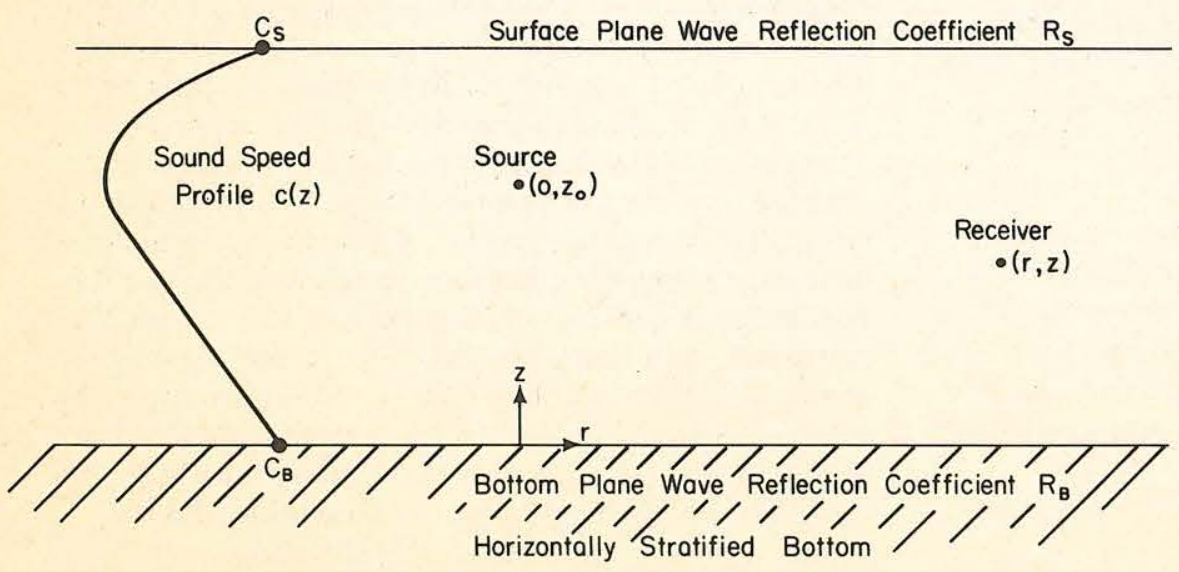

FIG. 1. Horizontally stratified ocean model. 


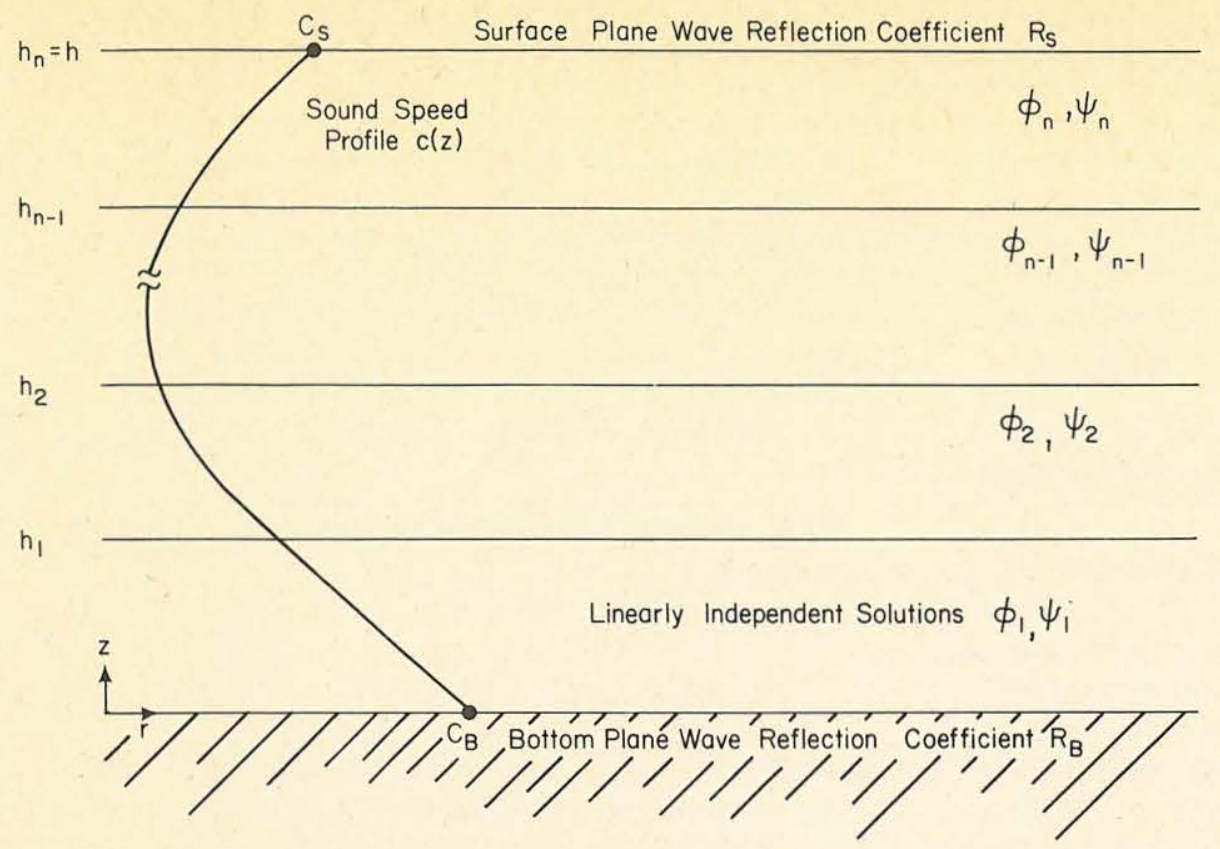

FIG. 2. Linearly independent solutions for construction of the Green's function.

with a half-space of sound speed $c_{s}$ bounding the surface. We therefore consider the following solutions (with $A$ and $B$ being arbitrary constants):

$$
\begin{aligned}
& p_{B}(z)=A\left[\exp \left(-i k_{B z} z\right)+R_{B}\left(k_{r}\right) \exp \left(i k_{B z} z\right)\right], \\
& p_{S}(z)=B\left[\exp \left(i k_{S z} z\right)+R_{S}\left(k_{r}\right) \exp \left(-i k_{S z} z\right)\right],
\end{aligned}
$$

where

$$
\begin{aligned}
& k_{B Z}=\left(k_{B}^{2}-k_{r}^{2}\right)^{1 / 2}, k_{S_{Z}}=\left(k_{S}^{2}-k_{r}^{2}\right)^{1 / 2}, \\
& k_{B}=\omega / c_{B}, \text { and } k_{S}=\omega / c_{S} .
\end{aligned}
$$

The conditions in Eq. (8) can then be written as

$$
\begin{aligned}
& \gamma_{B}=\frac{p_{B}}{p_{B}^{\prime}}=\frac{i\left(1+R_{B}\right)}{k_{B z}\left(1-R_{B}\right)}, \text { at } z=0, \\
& \gamma_{S}=\frac{p_{S}}{p_{S}^{\prime}}=\frac{-i}{k_{S z}}\left[\frac{1+R_{S} \exp \left(-2 i k_{S z} h\right)}{1-R_{S} \exp \left(-2 i k_{S z} h\right)}\right], \text { at } z=h .
\end{aligned}
$$

Bucker,${ }^{9}$ in constructing a normal mode solution for an inhomogeneous ocean, used a different approach to arrive at the same result for incorporating the effect of the boundaries through plane-wave reflection coefficients. He inserted a thin isovelocity layer of speed $c_{S}$ at the surface and a layer of speed $c_{B}$ at the bottom. Each layer then contained up-and down-going plane waves related by plane-wave reflection coefficients. He then shrank the layers to zero thickness at the end of the calculation. In any case, the key point is that the effect of the boundaries $c$ in be incorporated into the theory in an exact manne:- using plane-wave reflection coefficients even for the case of an inhomogeneous ocean.

Let us now address the problem of constructing $p_{B}$ and $p_{s}$. In general, $c(z)$ has a form which does not allow single functional forms of $p_{B}$ and $p_{S}$ to hold throughout the water column. However let us assume that $c(z)$ can be decomposed into segments within each one of which $p_{B}$ and $p_{S}$ can be expressed as a linear combination of known analytic, linearly independent solutions of the homogeneous version of Eq. (5). The solutions $p_{B}$ and $p_{S}$ are then given by (c.f. Fig. 2)

$$
p_{B}(z)=\left\{\begin{array}{cl}
A_{1 B} \phi_{1}(z)+B_{1 B} \psi_{1}(z), & 0 \leqslant z \leqslant h_{1}, \\
A_{2 B} \phi_{2}(z)+B_{2 B} \psi_{2}(z), & h_{1} \leqslant z \leqslant h_{2}, \\
\cdot & \\
\cdot & \\
A_{n B} \phi_{n}(z)+B_{n B} \psi_{n}(z), & h_{n-1} \leqslant z \leqslant h,
\end{array}\right.
$$

$$
p_{S}(z)=\left\{\begin{array}{cl}
A_{1 S} \phi_{1}(z)+B_{1 S} \psi_{1}(z), & 0 \leqslant z \leqslant h_{1}, \\
A_{2 S} \phi_{2}(z)+B_{2 S} \psi_{2}(z), & h_{1} \leqslant z \leqslant h_{2}, \\
\cdot & \\
\cdot & \\
A_{n S} \phi_{n}(z)+B_{n S} \psi_{n}(z), & h_{n-1} \leqslant z \leqslant h .
\end{array}\right.
$$

The coefficients $A_{i B}$ and $B_{i B}(i=1, \ldots, n)$, are obtained to within a multiplicative constant by imposing the conditions of continuous pressure and normal particle velocity at the segment interfaces and the impedance relation $(10 \mathrm{a})$ at the bottom. Similarly the coefficients $A_{i S}$ and $B_{i S}(i=1, \ldots, n)$ are obtained to within a multiplicative constant by imposing continuity conditions at the segment interfaces and the impedance relation (10b) at the surface. In each case, the multiplicative constant (i.e., one of the coefficients) is arbitrary, and therefore we have $2 n-1$ unknowns and $2 n-1$ equations. Using Eq. (10) we can relate $B_{1 B} / A_{1 B}$ to $R_{B}$ and $B_{n S} / A_{n S}$ to $R_{S}$ :

$$
\frac{B_{1 B}}{A_{1 B}}=\frac{i\left(1+R_{B}\right) \phi_{1}{ }^{\prime}(0)-k_{B z}\left(1-R_{B}\right) \phi_{1}(0)}{k_{B z}\left(1-R_{B}\right) \psi_{1}(0)-i\left(1+R_{B}\right) \psi_{1}^{\prime}(0)},
$$


The depth-dependent Green's function $g$ is then constructed by selecting the appropriate solutions in Eq. (11) depending upon the positions of $z$ and $z_{0}$ in the water column and combining them according to the formula in Eq. (7). Finally, the pressure field $G$ is obtained by taking the Hankel transform in $k_{r}$ of $g$.

\section{THE PRINCIPLE IN MEASURING THE PLANE- WAVE REFLECTION COEFFICIENT OF THE OCEAN BOTTOM}

In the previous section we saw that from knowledge of the sound speed structure in the water column and the plane-wave reflection coefficients of the boundaries, we could compute the pressure field $G$ due to a point source by taking the Hankel transform in horizontal wavenumber of the appropriate depth-dependent Green's function $g$. On the other hand, knowledge of the pressure field as a function of range at fixed $z$ and $z_{0}$ would allow us to compute the Green's function by taking the transform of $G$ in $r$ :

$$
g\left(k_{r}, z, z_{0}\right)=\int_{0}^{\infty} r d r J_{0}\left(k_{r} r\right) G\left(r, z, z_{0}\right) .
$$

It is the latter problem, namely that of inferring properties of the waveguide from measurements of the field, which we wish to focus upon here. In particular, we would like to determine the plane-wave reflection coefficient of the ocean bottom. The notion of inferring the reflective properties of the bottom by using the exact Hankel transform relationship between $g$ and $G$ was suggested by Stickler. ${ }^{4}$ However, in dealing with the case of an inhomogeneous ocean, he defined a reflection coefficient which is not the plane-wave reflection coefficient. On the other hand, we show explicitly that knowledge of $g$ can be used to determine the planewave reflection coefficient of the bottom even when the measurements are made in an inhomogeneous ocean.

In the previous section we saw that, for a particular sound speed profile, we could calculate $g$ once the coefficients $A_{i B}, B_{i B}, A_{i s}$, and $B_{i s}(i=1, \ldots, n)$ were known. These coefficients were determined by imposing continuity conditions at the segment interfaces and impedance relations at the boundaries. If we also know $g$ at some specified $z$ and $z_{0}$, then we have an additional constraint which eliminates the need for one of the boundary (or continuity) conditions. In fact we can consider one of the conditions as an unknown and solve for it. Specifically if we take the bottom impedance relation as our unknown, we can then determine $R_{B}$ by inverting Eq. (12a):

$R_{B}=\frac{\left(B_{1 B} / A_{1 B}\right)\left[k_{B z} \psi_{1}(0)-i \psi_{1}^{\prime}(0)\right]-i \phi_{1}^{\prime}(0)+k_{B z} \phi_{1}(0)}{\left(B_{1 B} / A_{1 B}\right)\left[k_{B z} \psi_{1}(0)+i \psi_{1}^{\prime}(0)\right]+i \phi_{1}^{\prime}(0)+k_{B z} \phi_{1}(0)}$.

\section{MEASUREMENT OF THE PLANE-WAVE REFLECTION COEFFICIENT OF THE BOTTOM IN AN ISOVELOCITY OCEAN}

In the remainder of the paper we shall focus upon the case in which the ocean is characterized by a constant sound speed $c$. For simplicity we shall also neglect the effect of the ocean surface. This condition is satisfied when the source output is sufficiently time-limited to allow us to gate out the surface reflection and multiple reflections within the water column. Thus our analysis applies to a single frequency component of a broadband pulse or more directly to a cw pulse of sufficient duration to establish steady-state conditions in the bottom returns.

In an isovelocity ocean, we have only the solutions $\phi_{1}$ and $\psi_{1}$ [c.f. Eq. (11)].

$$
\begin{aligned}
& \phi_{1}=\exp \left(-i k_{z} z\right), \\
& \psi_{1}=\exp \left(i k_{z} z\right),
\end{aligned}
$$

where $k_{z}=\left(k^{2}-k_{r}^{2}\right)^{1 / 2}$ and $k=\omega / c$. Neglecting the effect of the ocean surface is equivalent to imposing a radiation condition on $p_{S}$ at $\infty$. Therefore $A_{1 S}=0$ and $p_{B}$ and $p_{s}$ are given by

$$
\begin{aligned}
& p_{B}(z)=A_{1 B}\left[\exp \left(-i k_{z} z\right)+\left(B_{1 B} / A_{1 B}\right) \exp \left(i k_{z} z\right)\right], \\
& p_{S}(z)=B_{1 S} \exp \left(i k_{z} z\right) .
\end{aligned}
$$

The expression for $g$ then becomes [c.f. Eq. (7)]:

$$
\begin{aligned}
g\left(k_{r}, z, z_{0}\right)= & i k_{z}^{-1}\left\{\exp \left(i k_{z}\left|z-z_{0}\right|\right)\right. \\
& \left.+\left(B_{1 B} / A_{1 B}\right) \exp \left[i k_{z}\left(z+z_{0}\right)\right]\right\}, \quad 0 \leqslant z<\infty .
\end{aligned}
$$

Solving Eq. (17) for $\left(B_{1 B} / A_{1 B}\right)$ and substituting the re sult into Eq. (14), we obtain for the bottom reflection coefficient $R$ (dropping the subscript $B$ )

$$
\begin{aligned}
R\left(k_{r}\right)= & -\exp \left[-i k_{z}\left(z+z_{0}\right)\right]\left[\exp \left(i k_{z}\left|z-z_{0}\right|\right)\right. \\
& \left.+i k_{z} \int_{0}^{\infty} r d r J_{0}\left(k_{r} r\right) G\left(r, z, z_{0}\right)\right] .
\end{aligned}
$$

If the measured pressure field $p_{M}$ is that due to a point source of amplitude $p_{0}$ then Eq. (18) becomes

$$
\begin{aligned}
R\left(k_{r}\right)= & -\exp \left[-i k_{z}\left(z+z_{0}\right)\right]\left(\exp \left(i k_{z}\left|z-z_{0}\right|\right)\right. \\
& \left.+i k_{z} p_{0}^{-1} \int_{0}^{\infty} r d r J_{0}\left(k_{r} r\right) p_{M}\left(r, z, z_{0}\right)\right) .
\end{aligned}
$$

The total measured field can be decomposed into the incident field and reflected field

$$
p_{M}\left(r, z, z_{0}\right)=p_{I}\left(r, z, z_{0}\right)+p_{R}\left(r, z, z_{0}\right)
$$

where

$$
p_{I}\left(r, z, z_{0}\right)=p_{0}\left[\exp \left(i k R_{0}\right) / R_{0}\right],
$$

and $R_{0}=\left[r^{2}+\left(z-z_{0}\right)^{2}\right]^{1 / 2}$. But the Hankel transform of $p_{I}$ is given by

$$
H\left\{p_{I}\right\}=i k_{z}^{-1} p_{0} \exp \left(i k_{z}\left|z-z_{0}\right|\right),
$$

so that our expression for $R\left(k_{r}\right)$ now in terms of the reflected field only, becomes 


$$
\begin{aligned}
R\left(k_{r}\right)= & -i k_{z} p_{0}^{-1} \exp \left[-i k_{z}\left(z+z_{0}\right)\right] . \\
& \times \int_{0}^{\infty} r d r J_{0}\left(k_{r} r\right) p_{R}\left(r, z, z_{0}\right) .
\end{aligned}
$$

With a broadband source it may be possible to time resolve $p_{I}$ and $p_{R}$. However, with a cw pulse and nearbottom geometry, the steady state field will in general also include $p_{I}$. In that case, we shall assume that we know the source amplitude and experimental geometry with sufficient accuracy to remove $p_{I}$ from $p_{M}$

We note that the theoretical development can be phrased completely in terms of Fourier transforms which are interpreted physically as plane wave decompositions. ${ }^{10}$ Converting from polar to Cartesian coordinates, $p_{I}$ and $p_{R}$ become

$$
\begin{aligned}
p_{I}\left(x, y ; z, z_{0}\right) & \\
= & \frac{i p_{0}}{2 \pi} \int_{-\infty}^{\infty} \int_{-\infty}^{\infty} d k_{x} d k_{y}\left(k^{2}-k_{x}^{2}-k_{y}^{2}\right)^{-1 / 2} \\
\quad & \times \exp \left\{i\left[k_{x} x+k_{y} y+\left(k^{2}-k_{x}^{2}-k_{y}^{2}\right)^{1 / 2}\left|z-z_{0}\right|\right]\right\}, \\
p_{R}\left(x, y ; z, z_{0}\right) & \\
= & \frac{i p_{0}}{2 \pi} \int_{-\infty}^{\infty} \int_{-\infty}^{\infty} d k_{x} d k_{y}\left(k^{2}-k_{x}^{2}-k_{y}^{2}\right)^{-1 / 2} R\left(k_{x}, k_{y} ; k\right) \\
& \times \exp \left\{i\left[k_{x} x+k_{y} y+\left(k^{2}-k_{x}^{2}-k_{y}^{2}\right)^{1 / 2}\left(z+z_{0}\right)\right]\right\},
\end{aligned}
$$

where $r=\left(x^{2}+y^{2}\right)^{1 / 2}$ and $k_{r}=\left(k_{x}^{2}+k_{y}^{2}\right)^{1 / 2}$. We have expressed $p_{R}\left(x, y, z, z_{0}\right)$ as $p_{R}\left(x, y ; z, z_{0}\right)$ and $R\left(k_{x}, k_{y}, k\right)$ as $R\left(k_{x}, k_{y} ; k\right)$ in order to identify $z, z_{0}$, and $k$ as para meters rather than variables. Taking the inverse of Eq. (23b) we obtain

$$
\begin{aligned}
R\left(k_{x}, k_{y} ; k\right) \\
=(-i / 2 \pi)\left(k^{2}-k_{x}^{2}-k_{y}^{2}\right)^{1 / 2} p_{0}^{-1} \\
\quad \times \exp \left\{-i\left[\left(k^{2}-k_{x}^{2}-k_{y}^{2}\right)^{1 / 2}\left(z+z_{0}\right)\right]\right\} \\
\quad \times \int_{-\infty}^{\infty} \int_{-\infty}^{\infty} d x d y p_{R}\left(x, y ; z, z_{0}\right) \exp \left[-i\left(k_{x} x+k_{y} y\right)\right] .
\end{aligned}
$$

Virtually all existing techniques for estimating the reflection coefficient under the assumption of an isovelocity ocean and single bottom interaction rely on Eq. (22) and its inverse

$$
\begin{aligned}
p_{R}\left(r, z, z_{0}\right)= & i p_{0} \int_{0}^{\infty} k_{r} d k_{r} J_{0}\left(k_{r} r\right) \\
& \times R\left(k_{r}\right) k_{z}^{-1} \exp \left[i k_{z}\left(z+z_{0}\right)\right] .
\end{aligned}
$$

Conventional methods are based on the geometrical acoustics approximation of this integral, which yields ${ }^{10}$

$$
p_{R}\left(r, z, z_{0}\right) \approx R\left(\theta_{0}\right) p_{0} \exp \left[\left(i k R_{1}\right) / R_{1}\right],
$$

where $R_{1}=\left[r^{2}+\left(z+z_{0}\right)^{2}\right]^{1 / 2}$ and $\theta_{0}=\tan ^{-1}\left[r /\left(z+z_{0}\right)\right]$. This result states that the reflected field is a spherical wave emanating from the image source and multiplied by the reflection coefficient evaluated at the specular angle $\theta_{0}$ (c.f. Fig. 3). Thus $R$ can be measured as a function of angle by changing the source-receiver geometry and therefore the specular angle. But Stickler ${ }^{4}$ has shown that even for simple bottom types (e.g. isovelocity fluid

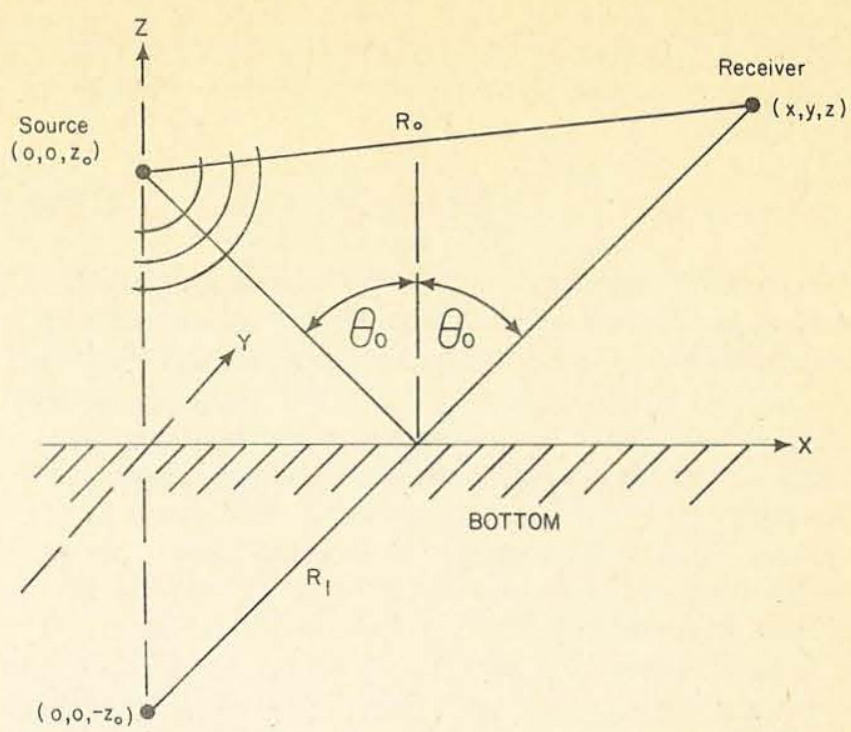

FIG. 3. The direct and specularly reflected arrivals.

half-space), the assumption of specular reflection can lead to erroneous and unphysical results. These include reflection coefficients which depend on sourcereceiver geometry and those with values greater than one for real angles. The need for more exact evaluation techniques has therefore recently given rise to several new approaches to the estimation problem. Schoenberg ${ }^{11}$ has adapted the Backus-Gilbert inversion method to this case. DiNapoli ${ }^{12,13}$ has applied the Fast Field Program (FFP) to the computation of the reflection coefficient from values of the reflected pressure field. The FFP algorithm relies on the asymptotic form for $J_{0}$

$$
J_{0}\left(k_{r} r\right) \sim\left(2 / \pi k_{r} r\right)^{1 / 2} \cos \left(k_{r} r-\pi / 4\right)
$$

which then allows the Hankel transform to be approximated in terms of a Fourier transform. An alternative is to use an algorithm, such as the one described in the companion paper, ${ }^{6}$ which does not require the use of this approximation. It is based on the "projectionslice" theorem for the two-dimensional Fourier transform. This theorem states that the one-dimensional Fourier transform of a projection of a function at some angle is a slice at the same angle of the two-dimensional Fourier transform of the function. In our case, where we have cylindrical symmetry, all of the projections are identical and the function is completely represented by a single projection. The application of the theorem then has a simple interpretation. From Eq. (24) let us consider the slice $k_{y}=0$ which corresponds to choosing the $x$ axis as the projection axis. Then we have (with $p_{0}=1$ )

$$
\begin{aligned}
R\left(k_{x}, 0 ; k\right)= & (-i / 2 \pi)\left(k^{2}-k_{x}^{2}\right)^{1 / 2} \exp \left[-i\left(k^{2}-k_{x}^{2}\right)^{1 / 2}\left(z+z_{0}\right)\right] \\
& \times \int_{-\infty}^{\infty} d x \rho\left(x ; z, z_{0}\right) \exp \left(-i k_{x} x\right)
\end{aligned}
$$

where

$$
\odot\left(x ; z, z_{0}\right)=\int_{-\infty}^{\infty} d y p_{R}\left(x, y ; z, z_{0}\right)
$$


is the projection of $p_{R}$ onto the $x$-axis. Again taking advantage of cylindrical symmetry, we can simplify Eq. (28b), so that

$$
\odot\left(x ; z, z_{0}\right)=2 \int_{0}^{\infty} d y p_{R}\left[\left(x^{2}+y^{2}\right)^{1 / 2} ; z, z_{0}\right] .
$$

Thus, the computation of the two-dimensional Fourier transform has been reduced to the computation of an area (the projection) and a one-dimensional Fourier transform. It may appear that we have complicated the problem by viewing the one-dimensional range integration in Eq. (22) as a two-dimensional integration in Cartesian coordinates. The power of this approach, however, lies in its computational advantages. The computation can be implemented by employing the algorithm described in detail in the companion paper. If $p_{R}$ is bandlimited, i.e., $g\left(k_{r} ; z, z_{0}\right)=0$ for $k_{r} \geqslant K$, and the samples of $P\left(x ; z, z_{0}\right)$ represent a finite-length sequence of length $\leqslant N \Delta x$, then the algorithm consists of a summation, a one-dimensional FFT, and multiplication by the depth-dependent factor:

$$
\begin{aligned}
\odot(n \Delta x)= & 2 \Delta y \sum_{l=0}^{\infty} p_{R}\left\{\left[(n \Delta x)^{2}+(l \Delta y)^{2}\right]^{1 / 2}\right\}, \\
R\left(m \Delta k_{r}\right)= & (-i / 2 \pi)\left[k^{2}-\left(m \Delta k_{r}\right)^{2}\right]^{1 / 2} \\
& \times \exp \left\{-i\left[k^{2}-\left(m \Delta k_{r}\right)^{2}\right]^{1 / 2}\left(z+z_{0}\right)\right\} \\
& \times \Delta x \sum_{n=0}^{N-1} \odot(n \Delta x) \exp (-i 2 \pi n m / N),
\end{aligned}
$$

where $\Delta k_{r}=2 \pi /(N \Delta x)$, and we require that $\Delta x \leqslant \pi / K$ and $\Delta y \leqslant 2 \pi / K$ by virtue of the sampling theorem.

In fact $p_{R}$ is not bandlimited since $g$ is given by

$$
g\left(k_{r} ; z, z_{0}\right)=R\left(k_{r}\right) B\left(k_{r} ; z, z_{0}\right),
$$

where

$$
\begin{aligned}
B\left(k_{r} ; z, z_{0}\right)= & {\left[i /\left(k^{2}-k_{r}^{2}\right)^{1 / 2}\right] } \\
& \times \exp \left[i\left(k^{2}-k_{r}^{2}\right)^{1 / 2}\left(z+z_{0}\right)\right], \quad 0 \leqslant k_{r}<k, \\
B\left(k_{r} ; z, z_{0}\right)= & {\left[1 /\left(k_{r}^{2}-k^{2}\right)^{1 / 2}\right] } \\
& \times \exp \left[-\left(k_{r}^{2}-k^{2}\right)^{1 / 2}\left(z+z_{0}\right)\right], \quad k<k_{r}<\infty .
\end{aligned}
$$

Using the relationship $k_{r}=k \sin \theta$, where $\theta$ is the angle of incidence, we see that the image source injects into the medium plane waves at both real $\left(0 \leqslant k_{r}<k\right)$ and complex $\left(k<k_{r}<\infty\right)$ angles of incidence. The former are pure propagating plane waves with angles $0 \leqslant \theta<\pi / 2$ while the latter are propagating waves in $x$ and $y$ and exponentially damped in $z$ and therefore called inhomogeneous plane waves. They can be represented in terms of complex angles $\theta=\pi / 2-i \alpha(\alpha>0)$. The function $|B|$ is sketched in Fig. 4. For real angles it has an algebraic dependence on $k_{r}$. For complex angles $|B|$ also exhibits an exponential behavior with a decay rate that depends on source/receiver height and frequency. At a fixed frequency, as the distance of source and/or receiver from the boundary increases, $|B|$ in the inhomogeneous wave region decreases. If the source and receiver are on the boundary, then the dependence of $|B|$ on $k_{r}$ is algebraic in both angular re-

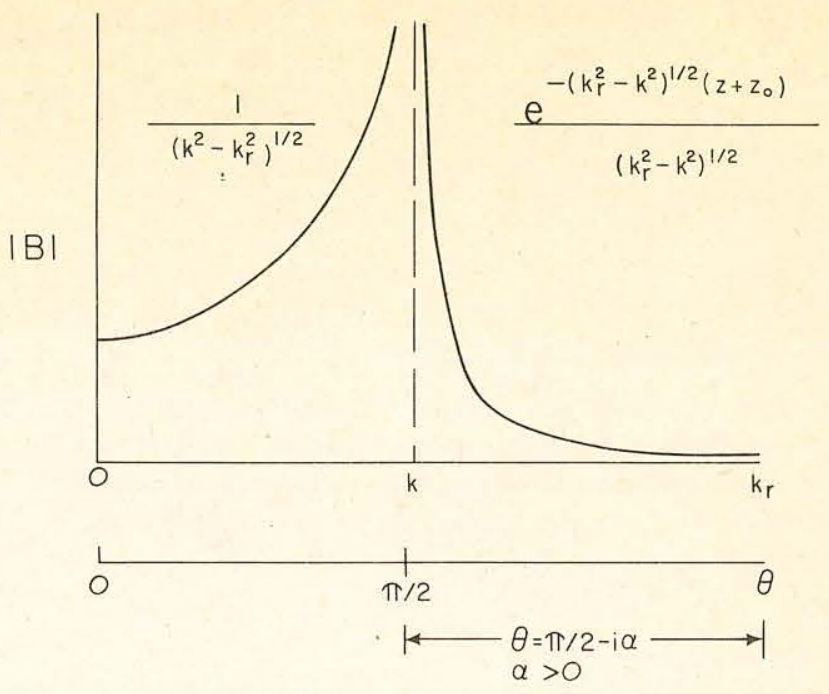

FIG. 4. The function $|B|$.

gions. Based on the behavior of $|B|$, it has been shown ${ }^{2}$ that a necessary, but not sufficient, condition for neglecting the inhomogeneous wave contribution is $(\lambda=2 \pi / k)$

$$
z+z_{0} \gg \lambda /(2 \sqrt{2} \pi) .
$$

These results are consistent with the geometrical acoustics approximation in which source and receiver are many wavelengths from the boundary and the reflection of inhomogeneous waves is neglected.

Let us now discuss the behavior of $R\left(k_{r}\right)$. By virtue of energy conservation, ${ }^{2}|R|$ is bounded by unity for real angles but can be unbounded for complex angles $\theta=\pi / 2-i \alpha(\alpha>0)$. In fact poles arise in $R$ when normal modes are excited in slow speed regions of the bottom via inhomogeneous -propagating wave conversion. An example of such a region occurs in surficial sediments, where the sound speed may decrease by about $1 \%-3 \%$ relative to the speed in water. In that case poles may appear in a narrow region $k<k_{r}<k(1-\epsilon)^{-1}$, where $\epsilon$ is the fractional drop in speed. With increasing depth, the sound speed is usually assumed to be monotonically increasing. Thus, in most cases we expect the poles to occur in the vicinity of the water wavenumber.

We have seen that determining whether or not $p_{R}$ is bandlimited is not generally straightforward. The properties of $g$ depend on the experimental frequency and geometry and on the characteristics of the bottom. In the situation where the combined source/receiver height is many wavelengths and there are no slow speed regions in the bottom, we can say that to a very good approximation $g \approx 0$ for $k_{r}>k$. Then the sampling theorem requires that we sample the field at least every halfwavelength. If there is a slow speed region in the surficial sediments, we must then sample at a somewhat higher rate corresponding to the case $g \approx 0$ for $k_{r}>k(1-\epsilon)^{-1}$. If the poles of $R$ are concentrated near $k$ but the source/receiver height is less than a wavelength, the sampling rate must be increased substantially above the half-wavelength value. 
In addition to the assumption that $p_{R}$ is bandlimited, the algorithm has the requirement that the samples of $\rho\left(x ; z, z_{0}\right)$ represent a finite-length sequence of length $\leqslant N \Delta x$. But $p_{R}$ is in general not range-limited, and therefore the finite sequence is obtained by truncating the pressure field at some range $R_{\max }$. This corresponds to applying a circularly symmetric window $w(r)$ such that $w(r)=0$ for $r \geqslant R_{\max }$. The algorithm therefore yields an estimate $\tilde{R}$ of the reflection coefficient where [c.f., Eqs. (24) and (28)]

$$
\begin{aligned}
\tilde{R}\left[\left(k_{x}^{2}+k_{y}^{2}\right)^{1 / 2} ; k\right]= & \frac{-i}{2 \pi}\left(k^{2}-k_{x}^{2}-k_{y}^{2}\right)^{1 / 2} \\
& \times \exp \left[-i\left(k^{2}-k_{x}^{2}-k_{y}^{2}\right)^{1 / 2}\left(z+z_{0}\right)\right] \\
& \times \int_{-\infty}^{\infty} \int_{-\infty}^{\infty} d x d y p_{R}\left[\left(x^{2}+y^{2}\right)^{1 / 2} ; z, z_{0}\right] \\
& \times w\left[\left(x^{2}+y^{2}\right)^{1 / 2} \exp \left[-i\left(k_{x} x+k_{y} y\right)\right] .\right.
\end{aligned}
$$

Thus $\tilde{R}$ is the two-dimensional convolution of $R$ with the two-dimensional Fourier transform $W$ of the window:

$$
\tilde{R}\left[\left(k_{x}^{2}+k_{y}^{2}\right)^{1 / 2} ; k\right]=\frac{1}{2 \pi} R\left[\left(k_{x}^{2}+k_{y}^{2}\right)^{1 / 2} ; k\right] * W\left[\left(k_{x}^{2}+k_{y}^{2}\right)^{1 / 2}\right] .
$$

The convolution process degrades the quality of the results through the effects of wavenumber leakage and decreased resolution. The selection of a window which minimizes these effects is therefore an important step in the implementation of this technique. ${ }^{14}$ Incorporating the window, we find that Eq. (29) for the processing algorithm becomes

$$
\begin{aligned}
\tilde{\rho}(n \Delta x)= & 2 \Delta y \sum_{l=0}^{N_{y}} p_{R}\left\{\left[(n \Delta x)^{2}+(l \Delta y)^{2}\right]^{1 / 2}\right\} \\
& \times w\left\{\left[(n \Delta x)^{2}+(l \Delta y)^{2}\right]^{1 / 2}\right\} \\
\tilde{R}\left(m \Delta k_{r}\right)= & (-i / 2 \pi)\left[k^{2}-\left(m \Delta k_{r}\right)^{2}\right]^{1 / 2} \\
& \times \exp \left\{-i\left[k^{2}-\left(m \Delta k_{r}\right)^{2}\right]^{1 / 2}\left(z+z_{0}\right)\right\} \\
& \times \Delta x \sum_{n=0}^{N_{x}-1} \tilde{\rho}(n \Delta x) \exp \left(-i 2 \pi n m / N_{x}\right),
\end{aligned}
$$

provided $\Delta x \leqslant \pi / K$ and $\Delta y \leqslant 2 \pi / K$. Here $N_{x}$ and $\left(N_{y}+1\right)$ are the total number of samples in the $x$ and $y$ directions, respectively. In terms of the computed Green's function $\tilde{g}$, we have

$$
\begin{aligned}
\tilde{g}\left(m \Delta k_{r}\right)= & \frac{\Delta x}{2 \pi} \sum_{n=0}^{N_{x}-1} \tilde{\rho}(n \Delta x) \exp \left(-i 2 \pi n m / N_{x}\right), \\
\tilde{R}\left(m \Delta k_{r}\right)= & \frac{-i}{2 \pi}\left[k^{2}-\left(m \Delta k_{r}\right)^{2}\right]^{1 / 2} \exp \left\{-i\left[k^{2}-\left(m \Delta k_{r}\right)^{2}\right]^{1 / 2}\right. \\
& \left.\times\left(z+z_{0}\right)\right\} \tilde{g}\left(m \Delta k_{r}\right) .
\end{aligned}
$$

\section{A. A scaling property}

Under certain conditions our measurement technique exhibits the scaling property illustrated in Fig. 5. If the frequency $f$, source/receiver height $\left(z+z_{0}\right)$ and range aperture $R_{\max }$ in one experiment are appropriately scaled by the factor $\Delta$ in a second experiment, then the technique will yield identical estimates for the reflection coefficient. This result can be proved by using
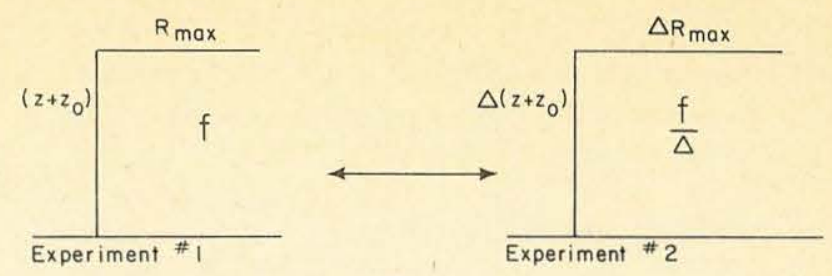

FIG. 5. A scaling property.

Eq. (32) with the assumption

$$
p_{R}\left(\frac{\left(x^{2}+y^{2}\right)^{1 / 2}}{\Delta}, \frac{z}{\Delta}, \frac{z_{0}}{\Delta}, \Delta k\right)=\Delta p_{R}\left[\left(x^{2}+y^{2}\right)^{1 / 2}, z, z_{0}, k\right]
$$

and the properties

$$
\begin{aligned}
& p_{R}(x, y) \stackrel{2-\mathrm{D} \mathrm{FT}}{\longrightarrow} g\left(k_{x}, k_{y}\right), \\
& p_{R}\left(\frac{x}{\Delta}, \frac{y}{\Delta}\right) \stackrel{2-\mathrm{D} \mathrm{FT}}{\longrightarrow} \Delta^{2} g\left(\Delta k_{x}, \Delta k_{y}\right), \\
& W\left(k_{x}, k_{y}\right)=\Delta^{2} W\left(\Delta k_{x}, \Delta k_{y}\right) .
\end{aligned}
$$

Using Eq. (23) and the transformation $k_{r}=k \sin \theta$, it can be shown that the assumption of Eq. (36) is valid only when $R$ is frequency-independent. This occurs when the bottom is perfectly reflecting $(R= \pm 1)$ or when it is a nonabsorbing isovelocity fluid ( $R$ is the Rayleigh reflection coefficient). A scaling property which is applicable for more general bottom types is currently
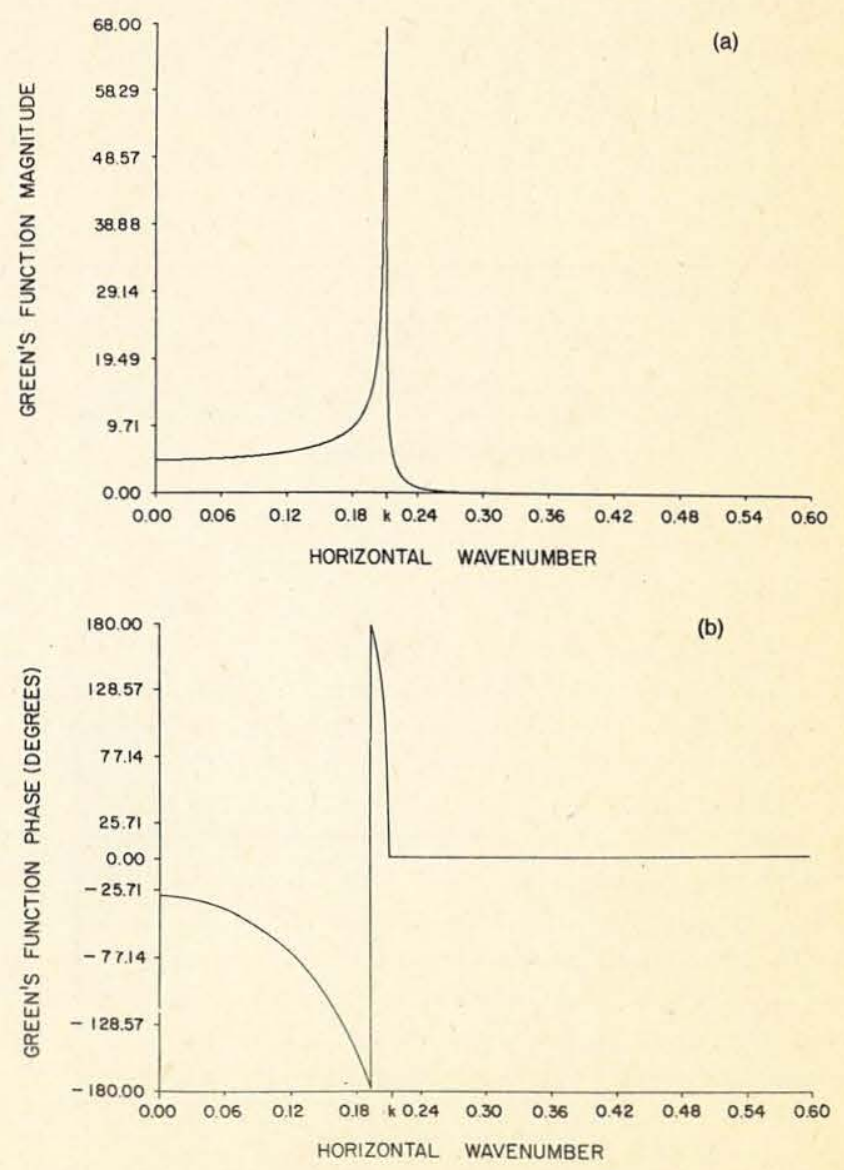

FIG. 6. Magnitude (a) and phase (b) of the exact Green's function for a perfectly reflecting hard bottom. 
being investigated.

To illustrate how the algorithm performs, we shall apply it to two specific bottom examples. These cases are discussed in detail in Ref. 14.

\section{B. Perfectly reflecting hard bottom}

In this section we shall apply our algorithm to the case of a perfectly reflecting hard bottom for which $g$ is given by Eq. (30) with $R=1$. The reflected pressure field is then given exactly by

$$
p_{R}=\frac{\exp \left\{i k\left[x^{2}+y^{2}+\left(z+z_{0}\right)^{2}\right]^{1 / 2}\right\}}{\left[x^{2}+y^{2}+\left(z+z_{0}\right)^{2}\right]^{1 / 2}}
$$

The physical parameters in our example are

$$
\begin{aligned}
& z=z_{0}=10 \mathrm{~m}, f=50 \mathrm{~Hz}, \quad c=1500 \mathrm{~m} / \mathrm{s}, \\
& k=0.20944 \mathrm{~m}^{-1} .
\end{aligned}
$$

The field was calculated using Eq. (38) over a range aperture $R_{\max }=2678.21 \mathrm{~m}$ at sample points such that

$$
K=0.6 \mathrm{~m}^{-1}, \Delta x=\pi / 0.6 \mathrm{~m}, \Delta y=\pi / 0.3 \mathrm{~m} .
$$

A circular Hanning window was applied to the points. The results for the magnitude and phase of the exact and computed Green's function are shown in Figs. 6
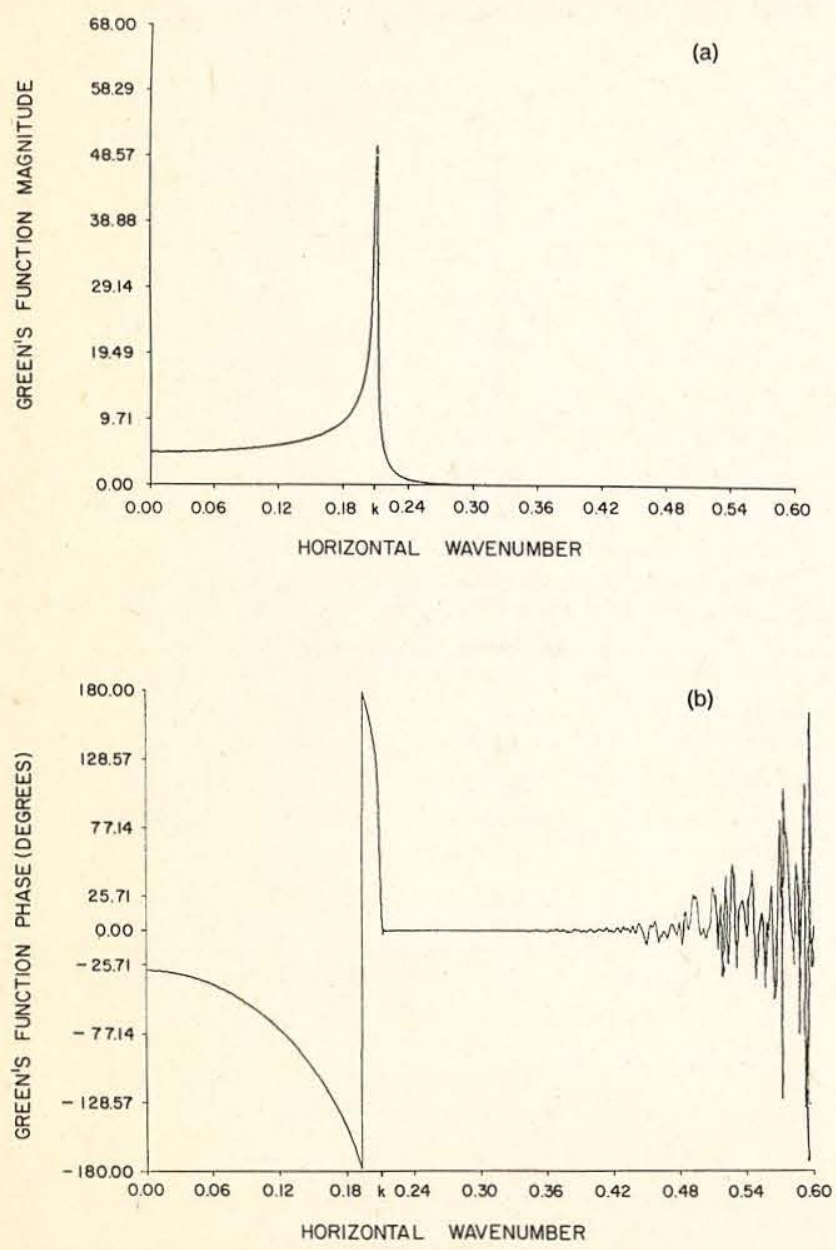

FIG. 7. Magnitude (a) and phase (b) of the computed Green's function for a perfectly reflecting hard bottom. and 7. The agreement in the magnitude is excellent for the entire range of $k_{r}$ considered. The phase result is excellent until $k_{r} \approx 0.42$, where it begins to deteriorate substantially. This is due to the fact that the Green's function is close to zero in that region, and therefore even small errors in the computed result can cause wide variations in phase. The computed results for the reflection coefficient are shown in Fig. 8. The behavior in the vicinity of the water wavenumber arises due to the singularity in $g$ at that point [c.f. Eq. (30)] and the corresponding steep slope in the vicinity of that point. If $\tilde{g}$ is even slightly misaligned in $k_{r}$ from $g$, then these oscillations will appear when we divide the two in order to obtain $\tilde{R}$. The degradation in the magnitude of $\tilde{R}$ for large $k_{r}$ arises due to the division of very small values of $\tilde{g}$ and $g$. The degradation in phase which appeared in $\tilde{g}$ naturally persists in the phase of $\tilde{R}$. These numerical deficiencies can be largely remedied by using double precision arithmetic $(\approx 13 \mathrm{sig}$ nificant figures on the Xerox Sigma 7). The substantially improved results in this case are shown in Fig. 9. One of the striking features of our method is that it yields results for the reflection coefficient in the inhomogeneous wave region. This is impossible with conventional methods which rely on the geometrical acoustics approximation and yield results only for real angles of incidence.


FIG. 8. Magnitude (a) and phase (b) of the computed reflection coefficient for a perfectly reflecting hard bottom.

Frisk et al.: Measuring the plane-wave reflection coefficient 

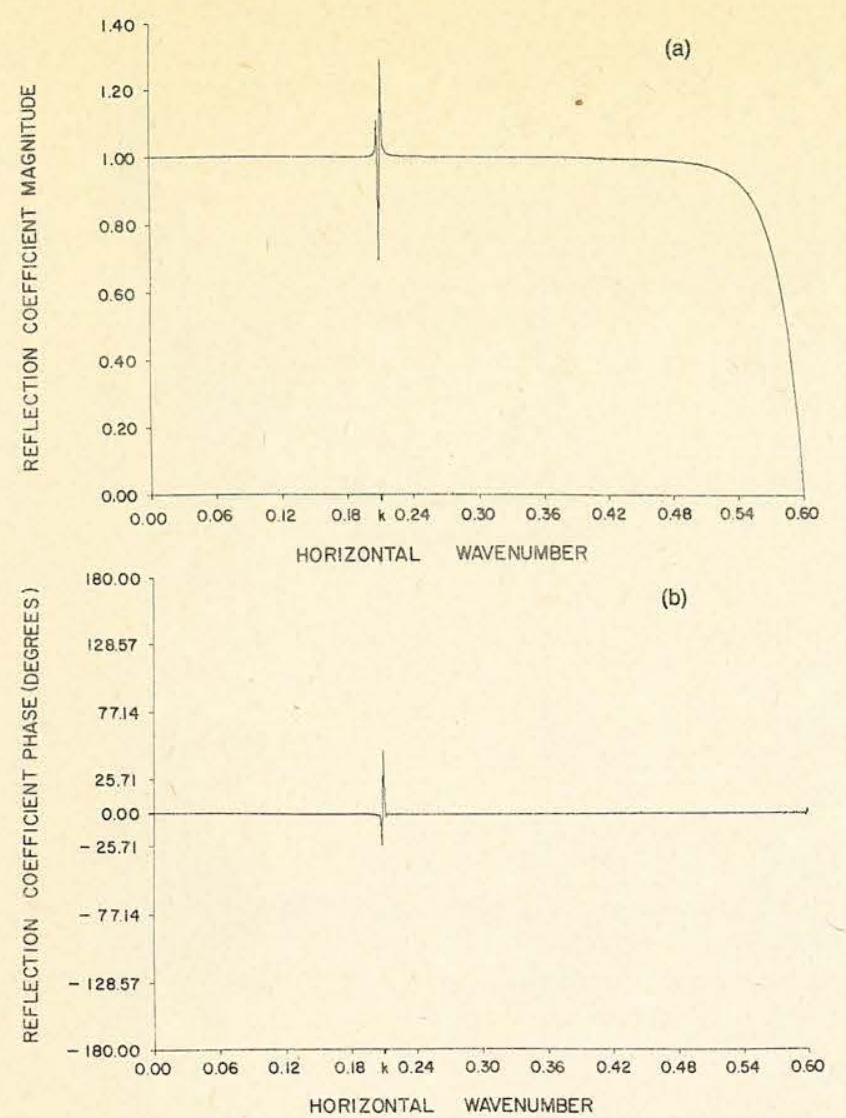

FIG. 9. Double precision magnitude (a) and phase (b) of the computed reflection coefficient for a perfectly reflecting hard bottom.
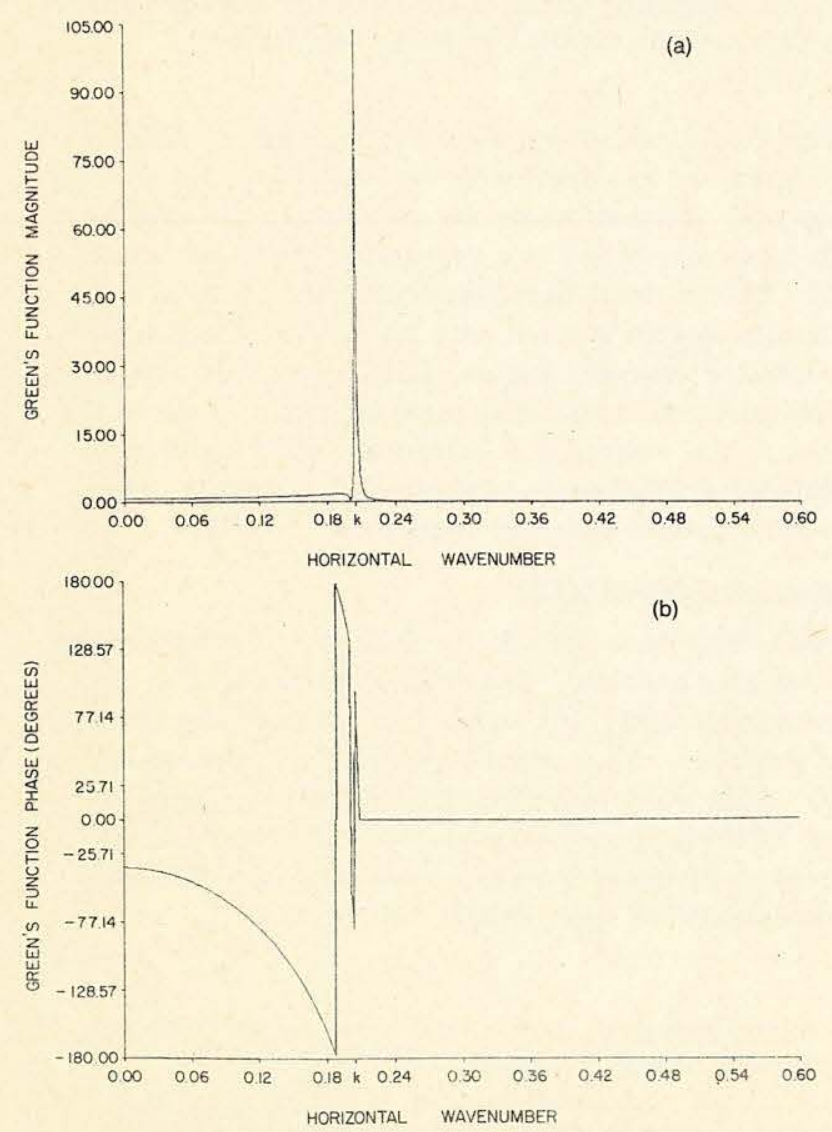

FIG. 10. Magnitude (a) and phase (b) of the exact Green's function for a slow isovelocity fluid bottom.

\section{Slow isovelocity fluid bottom}

Let us now consider the example of an isovelocity fluid bottom with density $\rho_{1}$ and sound speed $c_{1}$. The reflection coefficient is given by the Rayleigh reflection coefficient $t^{15}$

$$
R\left(k_{r}\right)=\frac{m\left(k^{2}-k_{r}^{2}\right)^{1 / 2}-\left(k_{1}^{2}-k_{r}^{2}\right)^{1 / 2}}{m\left(k^{2}-k_{r}^{2}\right)^{1 / 2}+\left(k_{1}^{2}-k_{r}^{2}\right)^{1 / 2}},
$$

where $m=\rho_{1} / \rho$ and $k_{1}=\omega / c_{1}$. We consider a slow bottom because there exists a simple approximate expression for the field in the geometrical acoustics approximation. In fact, surficial sediments may exhibit a drop in sound speed relative to the speed in water. The expression for $p_{R}$ is given by Eq. (26) which is valid in the entire specular angle domain for this case. The physical parameters in our example are

$$
\begin{aligned}
& z=z_{0}=10 \mathrm{~m}, f=50 \mathrm{~Hz}, \quad c=1540 \mathrm{~m} / \mathrm{s}, \\
& c_{1}=1509.2 \mathrm{~m} / \mathrm{s}=0.98 \mathrm{c}, \quad k=0.204 \mathrm{~m}^{-1}, \quad m=1.5 .
\end{aligned}
$$

We have chosen a $c_{1}$ which is $2 \%$ less than $c$ to simulate a typical drop in sound speed at the water-bottom interface. The aperture is $R_{\max }=2678.21 \mathrm{~m}$, and the sampling is that specified in Eq. (40). Again, a circular Hanning window was applied to the points. The results for the single-precision magnitude and phase of the exact and computed Green's function are shown in Figs.
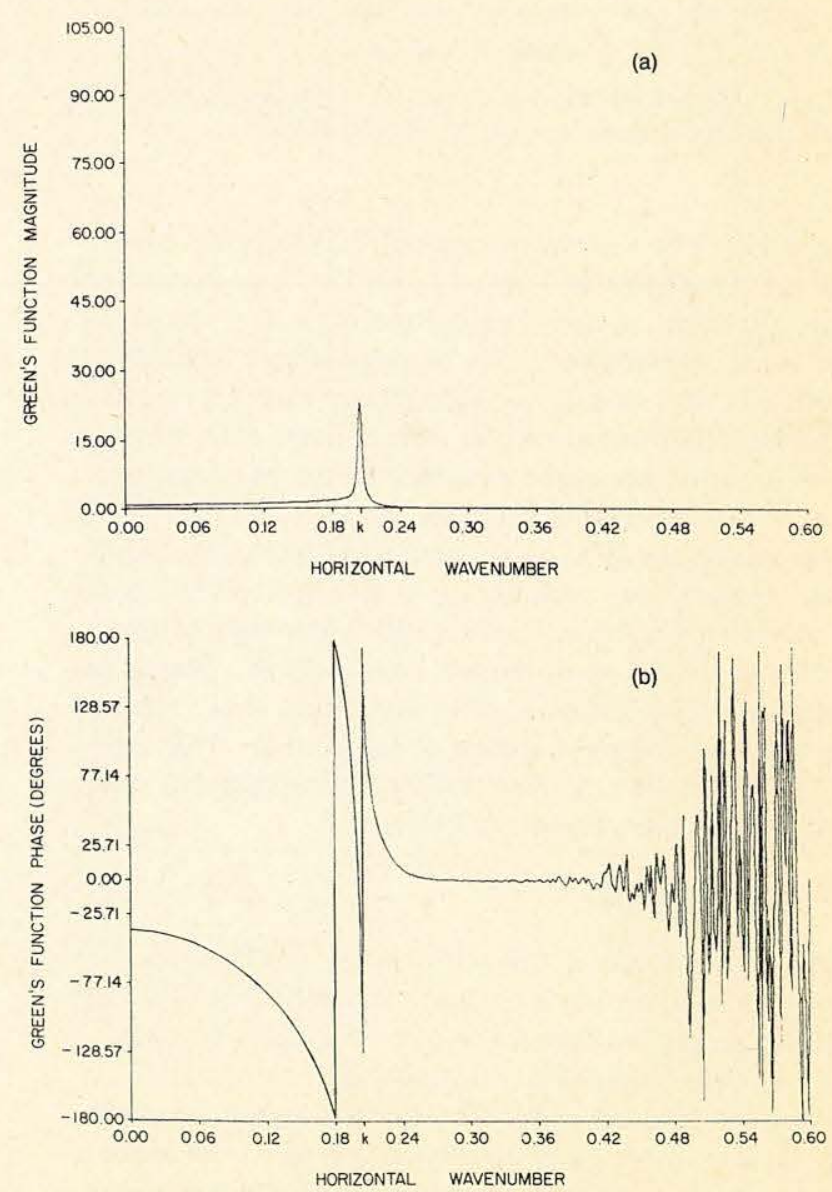

FIG. 11. Magnitude (a) and phase (b) of the computed Green's function for a slow isovelocity fluid bottom. 

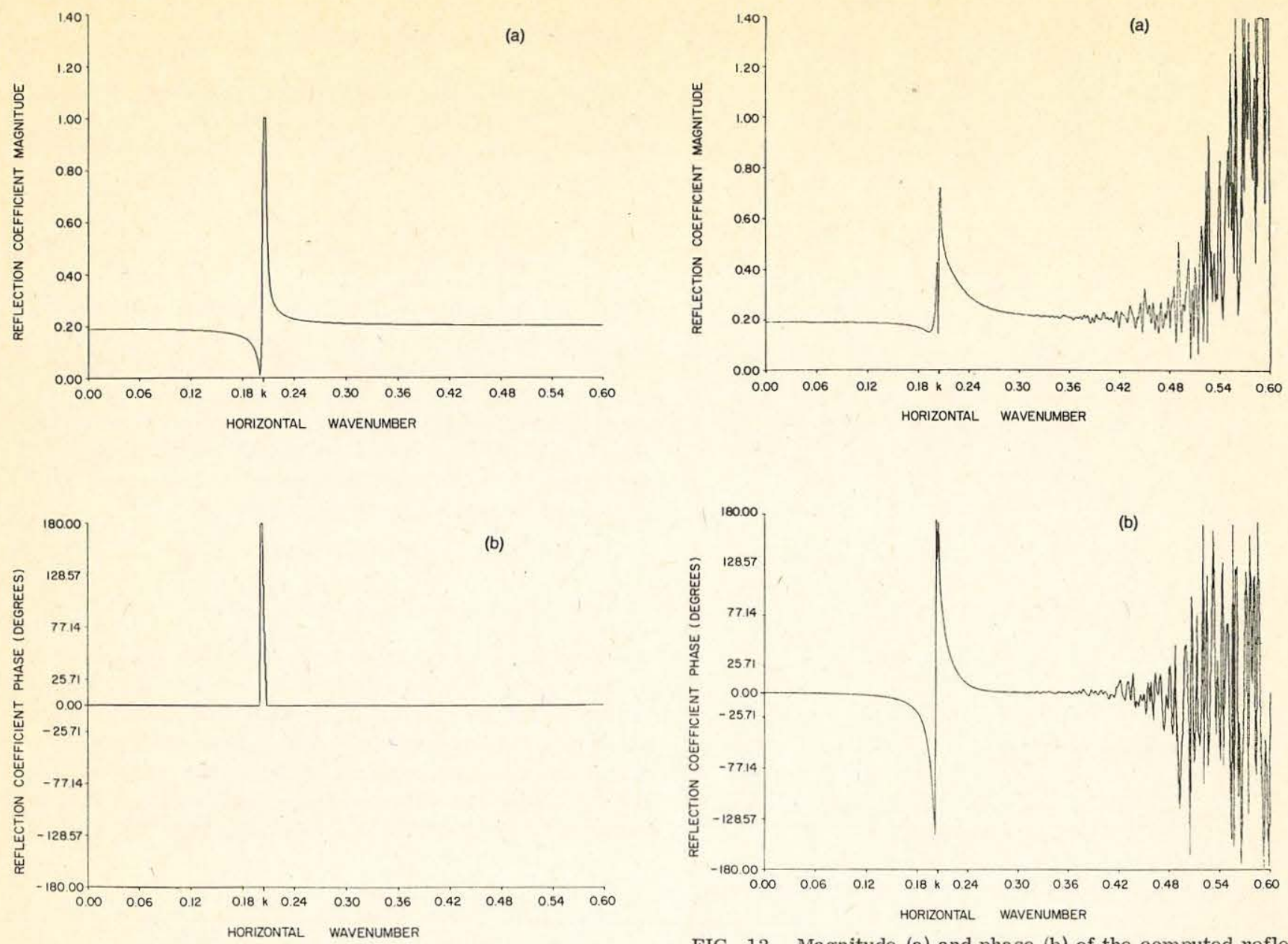

FIG. 12. Magnitude (a) and phase (b) of the exact reflection coefficient for a slow isovelocity fluid bottom.

10 and 11 . The exact and computed reflection coefficients are shown in Figs. 12 and 13. The numerical errors present in the hard bottom case are also evident here. However, in the regions where the numerical errors are small, the agreement is not as good as in the hard bottom example. We believe that, because of the relative accuracy obtained in the hard bottom case, the additional discrepancies here may be due to the geometrical acoustics approximation to the input field. In fact, our results could be used to assess the quality of this approximation. We again obtain good results in the inhomogeneous wave region. Thus, for real penetrable bottoms, this algorithm will yield information at complex angles of incidence. This may serve as a tool to provide substantial additional structural information about the bottom.

\section{CONCLUSION}

We have described a new technique for the measurement of the plane wave reflection coefficient of a horizontally stratified ocean bottom. It is based on the exact Hankel transform relationship between the reflection coefficient and the bottom reflected field due to a point source. The method employs a new algorithm for the numerical evaluation of the Hankel transform which is based on the "projection-slice" theorem

FIG. 13. Magnitude (a) and phase (b) of the computed reflection coefficient for a slow isovelocity fluid bottom.

for the two-dimensional Fourier transform. Although the algorithm was applied to the case of an isovelocity ocean, the general theory for measuring the plane wave reflection coefficient in a refracting ocean was developed. The technique provides information about the reflection coefficient not only for real incident angles, but also for complex angles, thus potentially providing substantial additional structural information about the bottom. The method was shown to yield excellent results with synthetically generated data for the cases of a hard bottom and slow isovelocity bottom.

\section{ACKNOWLEDGMENTS}

The authors are grateful to Robert P. Porter for suggesting this problem. David R. Martinez wishes to acknowledge WHOI for support of his graduate research assistantship. This paper is WHOI Contribution No. 4403. This work was supported in part by ONR Contract N00014-77-C-0196 and in part by the Advanced Research Projects Agency, monitored by ONR under Contract N00014-75-C-0951; NR049-328.

${ }^{1}$ L. M. Brekhovskikh, Waves in Layered Media (Academic, New York, 1960), pp. 15-61.

${ }^{2}$ G. V. Frisk, "Inhomogeneous waves and the plane-wave reflection coefficient," J. Acoust. Soc. Am. 66, 219-234 (1979). 
${ }^{3}$ K. E. Hawker and T. L. Foreman, "A plane wave reflection loss model based on numerical integration," J. Acoust. Soc. Am. 64, 1470-1477 (1978).

${ }^{4}$ D. C. Stickler, "The Principles Involved in the Measurement of Bottom Loss," Western Electric, NY, Report 72, R-245 (February 1972) (unpublished).

${ }^{5}$ D. C. Stickler, "Negative bottom loss, critical-angle shift, and the interpretation of the bottom reflection coefficient," J. Acoust. Soc. Am. 61, 707-710 (1977).

${ }^{6}$ A. V. Oppenheim, G. V. Frisk, and D. R. Martinez, "Computation of the Hankel transform using projections," J. Acoust. Soc. Am. 68, 523-529 (1980).

${ }^{7}$ P. M. Morse and H. Feshbach, Methods of Theoretical Physics (McGraw-Hill, New York, 1953), Part I, pp. 803828.

${ }^{8}$ Although both $g$ and $G$ are Green's functions, we shall refer to $g$ as the Green's function and $G$ as the pressure field.

${ }^{9}$ H. P. Bucker, "Sound Propagation in a Channel with Lossy
Boundaries," J. Acoust. Soc. Am. 48, 1187-1194 (1970).

${ }^{10}$ Reference 1, pp. 238-255.

${ }^{11} \mathrm{M}$. Schoenberg, "Nonparametric estimation of the ocean bottom reflection coefficient," J. Acoust. Soc. Am. 64, 11651170 (1978).

${ }^{12}$ F. R. DiNapoli, "The Inverse Fast Field Program (IFF P): An Application to the Determination of the Acoustic Parameters of the Ocean Bottom," Technical Memorandum No. 77160 , Naval Underwater Systems Center, New London, CT (5 August 1977) (unpublished).

${ }^{13}$ F. R. DiNapoli, "Fast Field Program for Multilayered Media," Report No. 4103, Naval Underwater Systems Center, Newport, RI (26 August 1971) (unpublished).

${ }^{14}$ D. R. Martinez, "Algorithm for Computation of the Acoustic Plane-Wave Reflection Coefficient of the Ocean Bottom," Electrical Engineer's thesis, Woods Hole Oceanographic Institute and Massachusetts Institute of Technology (June 1979).

${ }^{15}$ Reference 1 , pp. $15-22$. 


\section{MANDATORY DISTRIBUTION LIST}

\section{FOR UNCLASSIFIED TECHNICAL REPORTS, REPRINTS, AND FINAL REPORTS PUBLISHED BY OCEANOGRAPHIC CONTRACTORS \\ OF THE OCEAN SCIENCE AND TECHNOLOGY DIVISION \\ OF THE OFFICE OF NAVAL RESEARCH}

(REVISED NOVEMBER 1978)

1 Deputy Under Secretary of Defense

(Research and Advanced Technology)

Military Assistant for Environmental Science

Room 3D129

Washington, D.C. 20301

Office of Naval Research

800 North Quincy Street

Arlington, VA 22217

3 ATTN: Code 483

1 ATTN: Code 460

2 ATTN: 102B

1 CDR J. C. Harlett, (USN)

ONR Representative

Woods Hole Oceanographic Inst.

Woods Hole, MA 02543

Commanding Officer

Naval Research Laboratory

Washington, D.C. 20375

6 ATTN: Library, Code 2627
12 Defense Documentation Center Cameron Station

Alexandria, VA 22314

ATTN: DCA

Commander

Naval Oceanographic Office

NSTL Station

Bay St. Louis, MS 39522

1 ATTN: Code 8100

1 ATTN: Code 6000

1 ATTN: Code 3300

1 NODC/NOAA

Code D781

Wiscons in Avenue, N.W.

Washington, D.C. 20235 
UNCLASSIFIED $4 / 81$

SECURITY CLASSIFICATION OF THIS PAGE (When Data Entered)

\begin{tabular}{|c|c|}
\hline REPORT DOCUMENTATION PAGE & $\begin{array}{l}\text { READ INSTRUCTIONS } \\
\text { BEFORE COMPLETING FORM }\end{array}$ \\
\hline \begin{tabular}{l|l} 
1. REPORT NUMBER & 2. GOVT ACCESSION NO \\
WHOI $-81-24$ &
\end{tabular} & 3. RECIPIENT'S CATALOG NUMBER \\
\hline \multirow{2}{*}{$\begin{array}{l}\text { A TECHNIQUE FOR MEASLIRING THE PLANE-WAVE } \\
\text { REFLECTION COEFFICIENT OF THE OCEAN BOTTOM }\end{array}$} & $\begin{array}{l}\text { 5. TYPE OF REPORT A PERIOD COVERED } \\
\text { Technical }\end{array}$ \\
\hline & $\begin{array}{l}\text { 6. PERFORIAING ORG. REPORT NUMBER } \\
\text { WHOI Cont. \#4403 }\end{array}$ \\
\hline $\begin{array}{l}\text { 7. AUTHOR(o) } \\
\text { G.V. Frisk, A.V. Oppenheim, and D.R. Martinez }\end{array}$ & 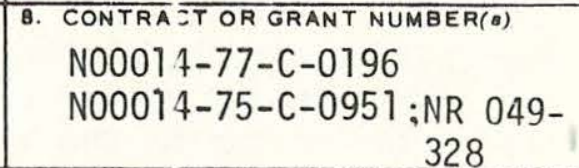 \\
\hline $\begin{array}{l}\text { 9. PERFORMING ORGANIZATION NAME AND ADDRESS } \\
\text { Woods Hole Oceanographic Institution } \\
\text { Woods Hole, Massachusetts } 02543\end{array}$ & $\begin{array}{l}\text { 10. PROGR A ELEMENT. PROJECT, TASK } \\
\text { AREA Q WORK UNIT NUMBERS } \\
\text { NR } 049-328\end{array}$ \\
\hline $\begin{array}{l}\text { 11. CONTROLLING OFFICE NAME AND ADDRESS } \\
\text { NORDA/ NATIONAL SPACE TECHNOLOGY LABORATORY } \\
\text { Bay St. Louis, MS } 39529\end{array}$ & \begin{tabular}{|l|l|} 
12. REPORT DATE \\
Apri 1 \\
13. NUMBEI OF PAGES \\
\end{tabular} \\
\hline \multirow[t]{2}{*}{ 14. MONITORING AGENCY NAME \& ADDRESS(It different from Controlling Offlice) } & $\begin{array}{l}\text { 15. SECURITY CLASS. (of this roport) } \\
\text { Unclassified }\end{array}$ \\
\hline & $\begin{array}{l}\text { 15a. DECLASSIFICATION/DOWNGRADING } \\
\text { SCHEDULEE }\end{array}$ \\
\hline \multicolumn{2}{|l|}{$\begin{array}{l}\text { 16. DISTRIBUTION STATEMEN T (of thi Roport) } \\
\text { Approved for public release; distribution unlimited. }\end{array}$} \\
\hline \multicolumn{2}{|l|}{ 17. DISTRIBUTION STATEMENT (of the abatract ontored in Block 20, If difforont from Roport) } \\
\hline
\end{tabular}

Reprinted from: J. Acoust. Soc. Am. 68, (2): 602-612 (August 1980).

19. KEY WORDS (Continue on reveras oide if neceseary and identify by block number)

1. Plane-wave reflection coefficient

2. Ocean bottom acoustics

3. Hankel transform

20. ABSTRACT (Continue on reverae efde if neceseary and identify by block number)

See Reverse 
A new technique for the measurement of the plane-wave reflection coefficient of a horizontally stratified ocean bottom is described. It is based on the exact Hankel transform relationship between the reflection coefficient and the bottom reflected field due to a point source. The method employs a new algorithm for the numerical evaluation of the Hankel transform which is based on the "projection-slice" theorem for the two-dimensional Fourier transform. The details of the algoithm are described in the companion paper. Although the algorithm is applied to the case of an isovelocity ocean, the general theory for measuring the plane-wave ref?ection coefficient in a refracting ocean is developed. The technique provides information about the reflection coefficient, not only for real incident angles, but also for complex angles, thus potentially providing substantial additional structural information about the bottom. The method is shown to yield excellent results with synthetically generated data for the cases of a hard bottom and slow isovelocity bottom. 


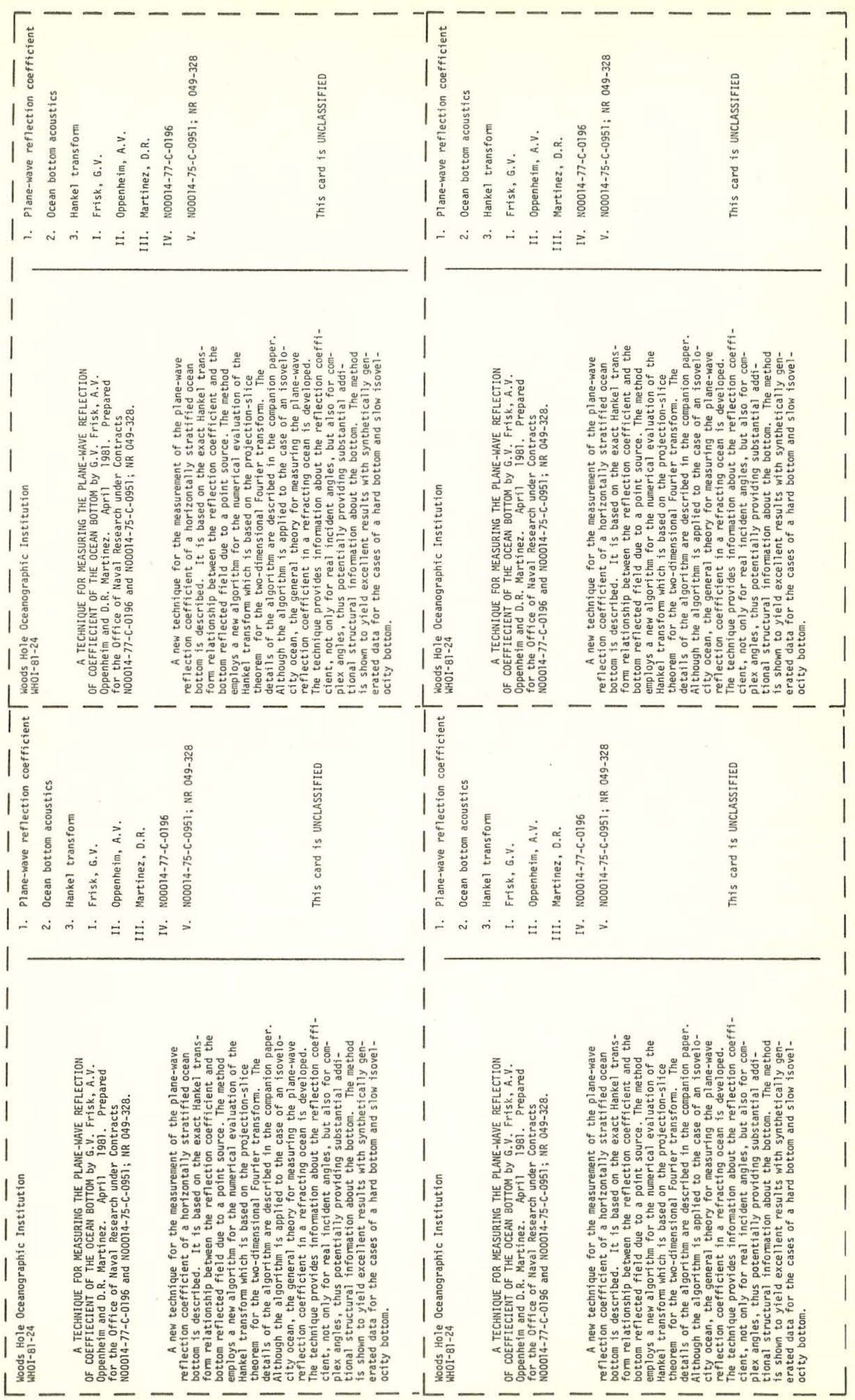

